ด

columns not guarantee that it will be understood and retained by students; in fact, Dochy et al in their systematic review find evidence that problem-based learning students retain their knowledge more effectively.

We were puzzled by Skokauskas assertion that problem-based learning 'assumes that students already are good problem-solvers'; in the courses we have experienced, problem solving is explicitly modelled as a skill to be developed by students. Certainly, the good group working skills needed for most working doctors cannot develop in a traditional lecture setting. Problem-based learning offers the opportunity to work in groups early and for individuals less 'keen' or 'capable' to identify their difficulties and reflect on them.

Last, Skokauskas worries that problembased learning students may be deprived of access 'to a particularly inspirational or charismatic professor'; this risk, we think, is mitigated by the fact that typical problem-based learning courses entail a three- or fourfold increase in hoursper-student of faculty contact. ${ }^{2}$

A recent systematic review of the effect of problem-based learning undergraduate courses on postgraduate competence suggests that problem-based learning trained doctors have stronger competencies in domains including coping with uncertainty, legal and ethical aspects of healthcare, communication skills, and self-directed continuing learning. ${ }^{3}$ These domains would seem to be of particular relevance to psychiatric practice, and we would encourage psychiatrists to get involved in the design and delivery of problem-based learning.

1 Dochy F, Segers M, van den Bossche P, Gijbels D. Effects of problem-based learning: a metaanalysis. Learn Instruct 2003; 5: 533-68.

2 Donner RS, Bickley H. Problem-based learning: an assessment of its feasibility and cost. Human Pathol 1990; 21: 881-5.

3 Koh GCH, Khoo HE, Wong ML, Koh D. The effects of problem-based learning during medical school on physician competency: a systematic review. Can Med Assoc J 2008; 178: 34 -41.

*Sheraz Ahmad Specialist Registrar in Psychiatry, Central North West London NHS FoundationTrust; Imperial College London; and London Deanery Fellow in Management and Leadership of Education in Psychiatry, email: sheraz77@hotmail.com Ben Braithwaite Clinical Teaching Fellow Department of Primary Care and Social Medicine, Imperial College London

doi: $10.1192 / p b .33 .6 .237 b$

\section{Clozapine monitoring - a gentler way}

Our learning disability team recently started clozapine for a young patient with treatment-resistant schizophrenia and a moderate intellectual disability. As treatment progressed, improvements were noted in the patient's positive symptoms and, perhaps more importantly, they started gaining some insight. However, although the patient credited the clozapine for this improvement, they were increasingly against the idea of regular blood monitoring. Continuing this monitoring in the community without their cooperation was not going to be possible. However, the team wanted the patient to continue to have the benefits the clozapine treatment was affording them.

Although venous blood sampling is the preferred method for monitoring neutrophil counts in adults, the team wondered whether our patient would be more amenable to blood monitoring if we used a capillary sampling technique favoured in paediatrics. There have been a few published papers looking at the variation in results for venous and capillary samples, but some studies had small sample sizes ${ }^{1}$ and others have included participants far younger than our patient. ${ }^{2}$

However, in a letter to the American Journal of Haematology, Schalk and colleagues $^{3}$ describe a study they undertook involving 421 adult patients, $70 \%$ of whom had a haematological disorder and $30 \%$ of whom were healthy volunteers; the age range was $18-61$ years. They concluded that capillary and venous absolute neutrophil counts correlate very well in adults. They also found there were no higher rates of infection using capillary sampling compared with venous sampling for their patients with neutropenia or agranulocytosis. They note that previous studies showed capillary samples had higher absolute neutrophil counts than venous samples but that this seemed to decrease with the increasing age of the cohort. In the 3 months to 14 years age group the variation between venous and capillary samples was $17.2 \%$, whereas in the $20-22$ years age group the difference in total leucocyte count was $9.2 \%$., 2 Both these studies are limited by small sample sizes ( $n=9$ and $n=24$ respectively).

The hospital pharmacy and clozapine monitoring service agreed for us to proceed with capillary sampling for our patient. The paediatric nurses provided practical advice for taking the sample. This included using petroleum jelly around the finger to make the blood form droplets as well as 'milking' the arm to reduce discomfort.

Although this method took more time than venous sampling, the patient was very happy with the finger-prick technique and volunteered to have a sample done with no extra effort. They were able to have this done in the community and were discharged

Although venous sampling remains the method of choice for monitoring patients on clozapine, we feel capillary sampling could be an option for patients who are unwilling to have the venous injection or where venous access proves difficult.

1 Yang ZW, Yang SH, Chen L, Qu J, Zhu J, Tang Z. Comparison of blood counts in venous, fingertip and arterial blood and their measurement variation. Clin Lab Haematol 2001; 23: 155-9.

2 Daae LNW, Hallerud M, Halvorsen S. A comparison between haematological parameters in'capillary' and venous blood samples from hospitalized children aged 3 months to 14 years. Scand J Clin Lab Invest 1991; 51: 651-4.

3 SchalkE, Scheinpflug K, Mohren M. Correlation of capillary and venous absolute neutrophil counts in adul thematological patients and normal controls. Am J Hematol 2008; 83: 605.

*Toral Thomas Specialty Registrar,

Cambridgeshire and Peterborough FoundationTrust, The Pines, Peterborough District Hospital, Peterborough PE3 6DA, email: toralthomas@ hotmail.com, Nauman Khalil Consultant Psychiatrist, Psychiatry of Learning Disabilities, Karen Wilkins Deputy Unit Manager, Psychiatry of Learning Disabilities, Cambridgeshire and Peterborough FoundationTrust

doi: 10.1192/pb.33.6.238

\section{Lost in translation}

A middle-aged male was transferred to our low secure forensic unit from a medium secure unit under hospital order with an accompanying restriction order. He had a diagnosis of paranoid schizophrenia, and was on antipsychotic treatment which caused a gradual mprovement of his psychotic symptoms. Although he was documented to have high premorbid functioning, ward staff attending the patient reported a marked cognitive decline in the weeks following his admission. He was referred for a neuropsychological assessment, which confirmed the cognitive decline and also detected lack of initiative and a reduction in processing speed. These were deemed not to be concordant with the negative symptoms of schizophrenia and an organic cause was suspected. An initial computed tomography scan and a subsequent magnetic resonance imaging (MRI) scan revealed mild cerebral atrophy.

Over subsequent months, his cognitive function continued to deteriorate and a further MRI scan showed a possible multiinfarct dementia; follow-up neurocognitive testing also suggested a further decline in cognitive abilities compared with the previous assessment. Therefore, we decided to refer him to the Burden Neurological Institute for a more detailed evaluation of the aetiology of his cognitive decline and with a view to organise future placement in the community. Burden Neurological Institute is a 